\title{
Selection and characterization of Aspergillus nidulans mutants involved in regulation of glycerol metabolism
}

\author{
DiRK H. A. HONDMANN and JAAP VISSER* \\ Department of Genetics, section Molecular Genetics, Agricultural University, Dreyenlaan 2, 6703 HA Wageningen, \\ The Netherlands
}

(Received 31 October 1991; accepted 7 January 1992)

\begin{abstract}
Pathway-specific induction plays an important role in Aspergillus nidulans when this fungus is grown on media containing glycerol as carbon source. To obtain mutants altered in regulation of glycerol metabolism, we used a glcB mutant strain, which is unable to grow on mixed carbon sources like D-glucose/glycerol as it accumulates G3P. Pseudorevertants, derived from this strain after mutagenesis as able to grow on D-glucose/glycerol, were analysed. In addition to glycerol kinase mutants and glycerol uptake mutants four new classes of mutants were found. Biochemical and genetic data indicate that the $\mathrm{glcH}$ mutants found are likely to lack a positive factor necessary for full induction of glycerol kinase and glycerol-3-phosphate dehydrogenase activities. The phenotype of a temperature-sensitive glcI mutant was found to be influenced by oxygen tension. Elevated levels of intracellular phosphatase were found in a glcL mutant. The $g l c K 1$ mutant had slow growth rates and higher levels of phosphofructokinase and fructose-6-phosphate reductase.
\end{abstract}

\section{Introduction}

In Aspergillus nidulans glycerol is catabolized by conversion to dihydroxyacetone phosphate (DHAP). After uptake, mediated by the $g l c C$ gene product, glycerol is first converted into glycerol 3-phosphate (G3P) by glycerol kinase (encoded by glc $A$ ). G3P is then oxidized to DHAP by a mitochondrially located FAD-dependent G3P dehydrogenase (encoded by $g l c B, g l c D$ and $g l c G$ ) (Arst et al., 1990; Hondmann et al., 1991). Dihydroxyacetone (DHA) and D-glyceraldehyde (D-GAD) catabolism is tightly linked to glycerol metabolism in $A$. nidulans. Both substrates are reduced intracellularly to glycerol, DHA by a constitutive NADPH-dependent reductase, and D-GAD by alcohol dehydrogenase I and by a constitutive NADPH-dependent reductase (Hondmann et al., 1991). The two NADPH-dependent reductase activities are attributed to a single enzyme that has been purified and characterized (Schuurink et al., 1990).

Two forms of regulation are found for both the glycerol kinase and the FAD-dependent G3P dehydrogenase activity. In the presence of glycerol, DHA and Dgalacturonate, pathway-specific induction occurs,

* Author for correspondence. Tel. 8370 82150; fax. 837083146 ; email GENETICA@RCL.WAU.NL.

Abbreviations: DHA, dihydroxyacetone; DHAP, dihydroxyacetone phosphate, G3P, glycerol 3-phosphate; D-GAD, D-glyceraldehyde. whereas in the presence of repressing carbon sources such as D-glucose, carbon catabolite repression is found (Hondmann et al., 1991).

A mutant of particular interest for the study of glycerol metabolism is the glcB33 mutant. This mutant has no G3P dehydrogenase (FAD) activity. As a result, G3P accumulates when mycelium of the mutant is transferred to media containing glycerol or DHA. Due to the accumulation of G3P the $g l c B 33$ mutant is unable to grow on media containing mixed carbon sources, e.g. Dglucose/glycerol or D-glucose/DHA.

We present here a study in which the glcB33 mutant is used to select pseudorevertants that grow on D-glucose/ glycerol. The nature of these pseudorevertants is discussed.

\section{Methods}

\footnotetext{
Materials. G3P dehydrogenase, lactate dehydrogenase, pyruvate kinase, enolase, glyceraldehyde-3-phosphate dehydrogenase, phosphoglycerate dehydrogenase, triosephosphate isomerase (all enzymes from rabbit muscle), glucose-6-phosphate dehydrogenase (from yeast), G3P, glycerate 2-phosphate, glycerate 3-phosphate, glycerate 2,3diphosphate, glyceraldehyde 3-phosphate, phosphoenolpyruvate, erythrose 4-phosphate, fructose 6-phosphate, 6-phosphogluconate, pnitrophenyl phosphate, ADP, ATP, NADH, NADPH, NADP ${ }^{+}$and $\mathrm{NAD}^{+}$were supplied by Boehringer. D-GAD, DHAP and MTT were from Sigma. DHA was from Serva. D-Galacturonic acid was obtained from Fluka. Sephadex G-25 was obtained from Pharmacia LKB. Other reagents were from Merck and were of analytical grade.
} 
Strains and culture conditions. Strain WG096 (yA2 pabaAl) was used as wild-type strain with respect to carbon source utilization. The glycerol non-utilizing strains WG196 (yA2 pabaAl glcA1), WG197 (yA2 pabaAl glcB33) WG372 (yA2 pantoB $100 \mathrm{glcC} 2)$, WG373 (yA2 pabaAl glcEl), WG374 (yA2 pabaAl glcFl), WG380 (biAl glcFl), WG381 (biAl glcEl), glcDI00 (pabaAl pantoA10 glcD100) and glcGl ( $y A 2$ pabaAl glcGl) were used. As tester strain for linkage group assignment WG202 (biAl acrAl meaB6 pyroA4 pAI sB3 nicB8 riboB2) was used. As a tester strain for the assignment of the glcK locus on chromosome II, WG362 (biAl acrAl wA7 palcAl riboE6 thiA4 ile $A 3$ cnxEl6 ygA6) was used. Genetic techniques were performed according to standard procedures (Pontecorvo et al., 1953; Clutterbuck, 1974). Culture conditions were described previously (Hondmann et al., 1991).

Growth tests were performed on minimal medium solidified with $1.2 \%(\mathrm{w} / \mathrm{v})$ agar and containing appropriate supplements, as described previously (Uitzetter $e t$ al., 1982). Carbon sources (pH 6) were added separately as membrane filter $(0 \cdot 2 \mu \mathrm{m}$, Schleicher and Schüll) sterilized solutions. The final concentrations were: glycerol, $100 \mathrm{~mm}$; DHA, $100 \mathrm{mM}$, D-galacturonic acid, $50 \mathrm{~mm}$; glucose/glycerol, $25 \mathrm{~mm} / 100 \mathrm{mM}$; glucose, $50 \mathrm{~mm}$; fructose, $50 \mathrm{~mm}$; glucose $/ D H A, 25 \mathrm{~mm} / 100 \mathrm{~mm}$; glucose/D-galacturonic acid, $25 \mathrm{~mm} / 50 \mathrm{mM}$; acetate, $100 \mathrm{mM}$; acetate/glycerol, $100 \mathrm{~mm} / 100 \mathrm{~mm}$. Microaerophilic growth conditions were obtained by incubating test plates in an 'exsicator' containing a BBL gas generator envelope for production of a reduced oxygen atmosphere, and which was used according to the manufacturer's instructions.

Mutagenesis. A suspension of approximately $10^{8}$ conidia from strain WG 197 ( $g l c B 33$ ) was irridiated for 1 min with a Philips TUV-lamp at a dose of $120 \mathrm{~J} \mathrm{~m}^{-2} \mathrm{~min}^{-1}$. This dose provides an optimum balance between the number of mutants that can be expected and background genetic damage (Bos, 1986).

Preparation of cell free extract. Cell free extracts were prepared according to Arst et al. (1990).

Enzyme assays and protein determination. Phosphatase activities were assayed in cell-free extracts essentially according to Caddick \& Arst (1986). Also a $10 \mathrm{~mm}$-glycylglycine/piperazine buffer was used to measure activities in one buffer system covering the range from $\mathrm{pH} 4 \cdot 0$ to $10 \cdot 0$. Phosphatase assays were performed at $30^{\circ} \mathrm{C}$.

Hexokinase (EC 2.7.1.1) was assayed in a reaction mixture containing $100 \mathrm{~mm}-\mathrm{Tris} / \mathrm{HCl} \quad(\mathrm{pH} 7.5), 10 \mathrm{mM}-\mathrm{MgCl}_{2}, 0.15 \mathrm{~mm}-$ $\mathrm{NADP}^{+}, 0.2 \mathrm{~mm}$-ATP, 1 U glucose-6-phosphate dehydrogenase, $4 \mathrm{~mm}$ glucose. Phosphoglucose isomerase (EC 5.3.1.9) was assayed in $40 \mathrm{~mm}$-triethanolamine $(\mathrm{pH} 7.6), 8 \mathrm{~mm}-\mathrm{MgCl}_{2}, 1 \mathrm{~mm}-\mathrm{NADP}{ }^{+}, 1 \mathrm{U}$ glucose-6-phosphate dehydrogenase, $2 \mathrm{~mm}$-fructose 6-phosphate. Phosphofructokinase (EC 2.7.1.11) was assayed in $50 \mathrm{mM}$-HEPES (pH 7.8), 2 mM- $\mathrm{MgCl}_{2}, 0.15 \mathrm{~mm}-\mathrm{NADH}, 1 \mathrm{~mm}-\mathrm{ATP}, 10 \mathrm{U}$ G3P dehydrogenase, $10 \mathrm{U}$ triose phosphate isomerase, $10 \mathrm{~mm}$-fructose 6phosphate. Fructose-1,6-diphosphate aldolase (EC 4.1.2.13) was assayed in $100 \mathrm{~mm}$-triethanolamine (pH 7.6), $10 \mathrm{~mm}$-EDTA, $0.2 \mathrm{mM}-$ erythrose 4-phosphate, 0.15 mM-NADH, 1 U G3P dehydrogenase, 10 $\mathrm{U}$ triose phosphate isomerase, $3 \mathrm{~mm}$-fructose 6-phosphate. Triose phosphate isomerase (EC 5.3.1.1) was assayed in $100 \mathrm{~mm}$-triethanolamine (pH 7.6), 0.15 mM-NADH, 1 U G3P dehydrogenase, $4 \mathrm{~mm}$ glyceraldehyde 3-phosphate. Glyceraldehyde-3-phosphate dehydrogenase (EC 1.2.1.13) was assayed in $100 \mathrm{mM}$-triethanolamine (pH 7.6), 1 mM-ATP, 1 mM-EDTA, 2 mM-MgSO, 0.15 mM-NADH, $20 \mathrm{U}$ phosphoglycerate kinase, $6 \mathrm{~mm}$-glycerate 3-phosphate. Phosphoglycerate kinase (EC 2.7.2.3) was assayed in 40 mM-triethanolamine (pH 7.6), 1 mM-EDTA, 0.15 mM-NADH, 1 mM-ATP, 2 mM-MgSO , $5 \mathrm{U}$ glyceraldehyde-3-phosphate dehydrogenase, $5 \mathrm{~mm}$-glycerate 3-phosphate. Phosphoglyceromutase (EC 2.7.5.3) was assayed in 100 mM-triethanolamine (pH 7.6), $1 \mathrm{~mm}-\mathrm{MgSO}_{4}, 0.15 \mathrm{~mm}-\mathrm{NADH}$,
0.6 mM-ADP, 0.12 mM-glycerate 2,3-diphosphate, $10 \mathrm{U}$ lactate dehydrogenase, $2 \mathrm{U}$ pyruvate kinase, $2 \mathrm{U}$ enolase, $5 \mathrm{~mm}$-glycerate 3phosphate. Enolase (EC 4.2.1.11) was assayed in $100 \mathrm{~mm}$-triethanolamine ( $\mathrm{pH} 7.6$ ), $4 \mathrm{~mm}-\mathrm{MgSO}_{4}, 0.15 \mathrm{~mm}-\mathrm{NADH}, 1 \mathrm{~mm}-\mathrm{ADP}, 20 \mathrm{U}$ lactate dehydrogenase, $3 \mathrm{U}$ pyruvate kinase, $1 \mathrm{~mm}$-glycerate 2phosphate. Pyruvate kinase (EC 2.7.1.10) was assayed in $100 \mathrm{~mm}$ triethanolamine ( $\mathrm{pH} 7.6$ ), $5 \mathrm{~mm}-\mathrm{MgCl}_{2}, 2 \mathrm{~mm}$-ADP, $0.15 \mathrm{~mm}-\mathrm{NADH}$, $13 \mathrm{U}$ lactate dehydrogenase, $10 \mathrm{mM}-\mathrm{KCl}, 10 \mathrm{~mm}-\left(\mathrm{NH}_{4}\right)_{2} \mathrm{SO}_{4}, 5 \mathrm{~mm}$ phosphoenolpyruvate. Fructose-1,6-diphosphatase (EC 3.1.3.11) was assayed in $50 \mathrm{~mm}$-Tris/ $\mathrm{HCl}$ (pH 8.0), $1 \mathrm{~mm}-\mathrm{EDTA}, 5 \mathrm{~mm}-\mathrm{MgCl}_{2}$, 0.4 mM-NADP+ 1 U glucose-6-phosphate dehydrogenase, $1 \mathrm{U}$ glucose6-phosphate isomerase, $2 \mathrm{~mm}$-fructose 1,6-diphosphate. Glucose-6phosphate dehydrogenase (EC 1.1.1.49) was assayed in $50 \mathrm{~mm}$ Tris/HCl (pH 8.0), 5 mM- $\mathrm{MgCl}_{2}, 0.4 \mathrm{~mm}-\mathrm{NADP}^{+}, 5 \mathrm{~mm}$-glucose 6phosphate. 6-Phosphogluconate dehydrogenase (EC 1.1.1.44) was assayed in $50 \mathrm{~mm}$-glycylglycine ( $\mathrm{pH} \mathrm{8.0)}, 0.4 \mathrm{~mm}-\mathrm{NADP}^{+}, 2 \mathrm{~mm}-6-$ phosphogluconate. NADP-dependent isocitrate dehydrogenase (EC 1.1.1.42) was assayed in $50 \mathrm{~mm}-\mathrm{HEPES}(\mathrm{pH} 7.5), 2 \mathrm{~mm}-\mathrm{MnSO}_{4}$, 0.2 mM-NADP ${ }^{+}, 2.5 \mathrm{mM}$-DL-isocitrate. NAD-dependent isocitrate dehydrogenase (EC 1.1.1.41) was assayed in $50 \mathrm{mM}$-HEPES ( $\mathrm{pH} 7.5$ ),

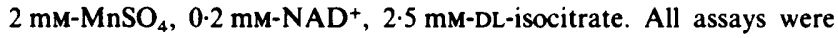
performed at $25^{\circ} \mathrm{C}$. One unit (U) of activity is expressed as $1 \mu \mathrm{mol}$ $\mathrm{NAD}(\mathrm{P}) \mathrm{H}$ formed or oxidized $\mathrm{min}^{-1}$.

The protein content and the activities of other enzymes were measured as described by Hondmann et al. (1991).

G3P determination. Small portions of frozen mycelium, to which $0.5 \mathrm{ml}$ double-distilled water and $50 \mu 160 \%(\mathrm{w} / \mathrm{v})$ perchloric acid were added, were ground up using a Elvejem tube. The suspension was incubated for $1 \mathrm{~h}$ on ice and then centrifuged $(10 \mathrm{~min}, 14000$ r.p.m., $4{ }^{\circ} \mathrm{C}$ ). The supernatant was neutralized by adding $8 \mathrm{M}-\mathrm{KOH}$. G3P content was determined in an enzymic assay containing $1 \mathrm{ml} 100 \mathrm{~mm}-$ glycine/500 mM-hydrazine (pH 9.6), 0.2 mM-NAD ${ }^{+}, 1$ U G3P dehydrogenase (rabbit muscle) and $50 \mu \mathrm{l}$ neutralized supernatant. G3P content is expressed in nmol ( $\mathrm{g}$ dry wt mycelium $)^{-1}$

\section{Results}

\section{Isolation and growth characteristics of glcB} pseudorevertants

After mutagenesis the conidiospore suspension was plated on 50 minimal medium agar Petri dishes containing D-glucose/glycerol $(50 \mathrm{~mm} / 100 \mathrm{mM})$ as carbon source. After incubation for $2 \mathrm{~d}$ at $37^{\circ} \mathrm{C}, 1400$ growing colonies were found. From these, 500 colonies were tested on a range of media. On glycerol and DHA testplates no colonies were found that grew as well as wild-type WG096, so we concluded that no $g l c B^{+}$ revertants were generated. It must be pointed out also that growth characteristics of the pseudorevertants selected tended to drift somewhat in successive tests, but we never observed wild-type growth on glycerol or DHA testplates. Of the pseudorevertants isolated, 20 were taken for further analysis.

\section{Genetic characterization of pseudorevertants}

The 20 pseudorevertants selected were used to form diploids with tester strain WG202, which has a marker on each chromosome. Diploids derived from each 
Table 1. Growth characteristics of $A$. nidulans pseudorevertants outcrossed from glcB33

Growth tests were performed at $37^{\circ} \mathrm{C}$ as described in Methods. ++++ , Good growth; +++ , moderate growth; ++ , poor growth, + , very poor growth; - , no growth. The number of mutations at a specific locus and the chromosome involved are also indicated.

\begin{tabular}{|c|c|c|c|c|c|c|c|c|}
\hline & Locus & glcA & glcC & glcH & glcI & glcK & glcL & WG096 \\
\hline \multicolumn{9}{|l|}{ Carbon source } \\
\hline Glucose & & $+++t$ & $+++t$ & ++++ & $+++t$ & +++ & +++ & ++++ \\
\hline Fructose & & +++ & +++ & $++t$ & +++ & +++ & +++ & $++t$ \\
\hline Acetate & & +++ & +++ & +++ & $\begin{array}{l}+++ \\
++t\end{array}$ & $\begin{array}{l}++ \\
++\end{array}$ & ++ & $\begin{array}{l}++t \\
+t+\end{array}$ \\
\hline Glycerol & & + & + & ++ & +++ & ++ & - & +++ \\
\hline DHA & & - & ++ & ++ & + & ++ & - & $++t$ \\
\hline D-Galacturonate & & + & +++ & ++ & +++ & ++ & - & +++ \\
\hline D-Glucose/glycerol & & $++t$ & $++t$ & $+t+$ & $++t$ & ++ & $+t+$ & $+t+$ \\
\hline D-Glucose/DHA & & $+t+t$ & $+t+t$ & $++t$ & $++t$ & +++ & ++ & $+t+t$ \\
\hline No. of pseudorevertants found & & 12 & 3 & 2 & 1 & 1 & 1 & \\
\hline Chromosome involved & & $\mathrm{V}$ & VI & V & III & II & $\mathbf{v}$ & \\
\hline
\end{tabular}

pseudorevertant and WG202 were haploidized to determine on which chromosome the new mutation was located and to separate it from the $g l c B$ mutation, provided that it is not located on chromosome I which contains $g l c B$. All the mutations could in this way be assigned to a particular chromosome. The outcrossed revertants were then phenotypically characterized. Table 1 lists the phenotype and genotype of the 20 outcrossed pseudorevertants and the number of pseudorevertants found to correspond to various loci.

Three pseudorevertants resembled the $\mathrm{glcC} 2$ mutant, both in linkage group allocation (chromosome VI) and in phenotype (Visser et al., 1988). Complementation analysis with the $g l c C 2$ mutant and with the $g l c E 1$ and $g l c F 1$ mutants (Hondmann et al., 1991), which are also on chromosome VI, revealed that these three pseudorevertants were mutated at the $g l c C$ locus.

Fifteen pseudorevertants had a mutation on chromosome V. The phenotype of 12 of these pseudorevertants was similar to that of the glcAl mutant, which is also on chromosome V (Payton, 1978). These 12 mutants failed to complement $g l c A l$ in both heterokaryons and diploids. The 12 pseudorevertants did complement nonallelic glycerol non-utilizing mutations in $g l c B, C, D, E, F$ and $G$. These 12 pseudorevertants are therefore considered to be mutated at the glc $A$ locus.

Of the three other pseudorevertants with a mutation on chromosome $\mathrm{V}$, two show phenotypical similarities to each other. The outcrossed pseudorevertants grew poorly on glycerol, DHA and D-galacturonate. This might be explained by leaky glycerol kinase mutations, as the glycerol kinase gene is also on chromosome V. However, the two outcrossed pseudorevertants complemented glcAl in heterokaryons as well as in diploids. Complementation with the other glycerol non-utilizing mutants revealed that these two pseudorevertants correspond to a new locus. Meiotic analysis of crosses of these two mutants showed very tight linkage, suggesting they are allelic. The mutants were designated $g l c H 1$ and $g l c H 2$. Diploids of $\mathrm{glcH} 1$ or $\mathrm{glcH} 2$ and the wild-type grew normally on glycerol, DHA and D-galacturonate, indicating that both mutations are recessive.

The fifteenth pseudorevertant with a mutation on chromosome $\mathrm{V}$ grew poorly on a wide range of carbon sources (over 40 different ones were tested), both in a $g l c B 33$ background as well as outcrossed from $g l c B 33$. The outcrossed mutant and the mutant in a $g l c B 33$ background did not grow on glycerol, DHA or Dgalacturonate. Complementation analysis with other glycerol non-utilizing mutants showed that this mutant does not correspond to any of the known glycerol mutations. This mutant was designated glcLl.

One pseudorevertant with a mutation on chromosome III was found. When outcrossed from $\mathrm{glcB33}$, the mutant grew like the wild-type WG096 on all carbon sources, except on DHA at $37^{\circ} \mathrm{C}$. This mutant complemented all glycerol non-utilizing mutants isolated thus far in $A$. nidulans. This new mutant was designated glcIl.

Pseudorevertant number 20 has a mutation on chromosome II. Its phenotypical characteristics resembled that of the $g l c L 1$ mutant, except that the outcrossed mutant did grow on glycerol, DHA and D-galacturonate, albeit much more slowly than the wild-type WG096. The mutant complemented all other glycerol non-utilizing mutants and was designated glcKI.

\section{Localization of the glcA, glcH and glcL genes on chromosome $V$}

Analysis of crosses of $g l c A l, g l c H 1, g l c H 2$ and $g l c L 1$ strains with strains carrying different markers on chromosome $\mathrm{V}$ (nicA, pdhB, hxA, acuG and riboD) showed that the $g l c A$ and $g l c H$ genes are located near the $a c u G$ locus. The glcL locus was found to be located 


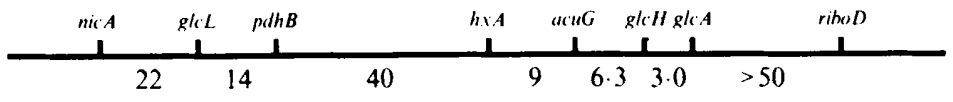

between the nic $A$ and $p d h B$ locus. A genetic map based on a number of crosses is shown in Fig. 1. Close proximity of $g l c A$ and $g l c H$ was confirmed by analysis of crosses of WG196 ( $\mathrm{glcAl}$ ) with $\mathrm{glcH} 1$ or $\mathrm{glcH} 2$ strains. The distance between the two genes was estimated to be approximately 3 centiMorgan.

\section{G3P accumulation}

Since in strains carrying the $g l c B 33$ mutation no growth is found on media containing mixed substrates like Dglucose and glycerol or D-glucose and DHA, presumably due to G3P accumulation, G3P levels were determined after transfer of mycelium to such media. The levels of G3P found are listed in Table 2.

Pseudorevertants lacking glycerol kinase would not be expected to accumulate G3P on media containing Dglucose/glycerol or D-glucose/DHA, and such accumulation did not occur in the glcAl mutant. Low levels of G3P were found in the $12 \mathrm{glcA}$ glcB33 mutants, of which a typical example is strain $g l c A 10$ glcB33.

A mutation inactivating the glycerol uptake system, as in the $g l c C 2$ mutant, would, in the presence of $g l c B 33$, not affect accumulation of G3P on media containing $D$ glucose/DHA, but would prevent such accumulation on $\mathrm{D}$-glucose/glycerol. This was indeed observed in the three glcCglcB33 mutants. Data obtained with glcC10glcB33 are given in Table 2 as an example.

Both $\mathrm{glcH} 1 \mathrm{glcB} 33$ and $\mathrm{glcH} 2 \mathrm{glcB} 33$ show low amounts of G3P on D-glucose/glycerol. The levels found are comparable to those found in the glycerol kinase mutant ( $g l c A l)$. G3P levels in the glcIl glcB33, and to a lesser extent those found in the glcL1 glcB33 mutant, are higher as in the wild-type WG096, but not as high as in the $g l c B 33$ strain. In the $g l c K 1$ glcB33 strain G3P levels are lower than those found in wild-type WG096, but not as low as those found in the glcAl strain.

\section{Glycerol kinase and mitochondrial G3P dehydrogenase activities in the glcH mutants}

glcH 1 and $\mathrm{glcH} 2$ strains were grown for $16 \mathrm{~h}$ at $37^{\circ} \mathrm{C}$ in minimal medium containing $50 \mathrm{mM}$-fructose, harvested by filtration and transferred for $6 \mathrm{~h}$ to fresh minimal medium containing $100 \mathrm{~mm}$-glycerol or $100 \mathrm{mM}$-DHA. Glycerol kinase and the flavin-dependent G3P dehydrogenase activities were measured. The activities found are
Fig. 1. Map of linkage group V of A. nidulans. Map distances are expressed in centiMorgans. For reported marker distances and marker nomenclature see Clutterbuck (1984).

\section{Table 2. G3P accumulation in glycerol mutants and in pseudorevertants}

Mycelia were grown for $16 \mathrm{~h}$ on minimal medium with fructose and then transferred for $8 \mathrm{~h}$ to fresh minimal medium containing $25 \mathrm{mM}$-D-glucose $/ 100 \mathrm{mM}$-glycerol or $25 \mathrm{mM}$-D-glucose $/ 100 \mathrm{mM}$ DHA. G3P determinations were done as described in Methods. Values given are means of two determinations. ND, Not determined.

\begin{tabular}{lcc}
\hline & \multicolumn{2}{c}{ G3P accumulation } \\
& [nmol (g dry wt mycelium) & \\
\cline { 2 - 3 } \multicolumn{1}{c}{ Strain } & D-Glucose/glycerol & D-Glucose/DHA \\
\hline WG096 & 14 & 20 \\
glcA1 & 4 & 3 \\
glcB33 & 51 & 90 \\
glcA10 glcB33 & 5 & 8 \\
glcC10 glcB33 & 3 & 88 \\
glcH1 glcB33 & 1 & ND \\
glcH2 glcB33 & 6 & ND \\
glcII glcB33 & 37 & 51 \\
glcK1 glcB33 & 7 & 15 \\
glcL1 glcB33 & 16 & 33 \\
\hline \hline
\end{tabular}

Table 3. Glycerol kinase and G3P dehydrogenase (FADdependent) activities in A. nidulans glcH mutants

Mycelia were grown for $16 \mathrm{~h}$ in minimal medium with fructose as carbon source and then transferred for $8 \mathrm{~h}$ to fresh minimal medium containing $100 \mathrm{~mm}$-glycerol, $100 \mathrm{~mm}$-DHA or $50 \mathrm{~mm}-\mathrm{D}$ fructose. Enzyme activities were determined as described in Methods. Data for WG096 were reported previously (Arst $e t$ al., 1990) but are included here to enable comparisons to be made.

\begin{tabular}{lcc}
\hline \hline & $\begin{array}{c}\text { Glycerol } \\
\text { kinase } \\
\text { [nmol } \text { min }^{-1}\end{array}$ & $\begin{array}{c}\text { G3P dehydrogenase } \\
\text { (FAD) }\end{array}$ \\
\hline glchl & & \\
Transfer to D-fructose & $\leq 2 \cdot 0$ & 11 \\
Transfer to glycerol & $\leq 2 \cdot 0$ & 20 \\
Transfer to DHA & $\leq 2 \cdot 0$ & 16 \\
glcH2 & & \\
Transfer to D-fructose & $\leq 2 \cdot 0$ & 9 \\
Transfer to glycerol & $\leq 2 \cdot 0$ & 5 \\
Transfer to DHA & $\leq 2 \cdot 0$ & 12 \\
WG096 & $\leq 2 \cdot 0$ & 10 \\
Transfer to D-fructose & 41 & 53 \\
Transfer to glycerol & 36 & 45 \\
Transfer to DHA & & \\
\hline \hline
\end{tabular}

shown in Table 3. In both of the glcH mutants glycerol kinase and G3P dehydrogenase activities are at uninduced or just slightly elevated levels upon transfer to inducing media containing glycerol or DHA. 
Temperature sensitive behaviour of the glcIl mutant

The glcll mutant grew on all carbon sources tested equally as well as the wild-type, except for DHA at $37^{\circ} \mathrm{C}$. A lag time of 3 to $5 \mathrm{~d}$ was observed before growth started on DHA at $37^{\circ} \mathrm{C}$. Once started, no growth differences compared to the wild-type were found. However, at lower temperatures $\left(20-30^{\circ} \mathrm{C}\right)$ no lag time was found.

When mycelia of $g l c I l$, grown in minimal medium containing $\mathrm{D}$-fructose as carbon source at either 30 or $37^{\circ} \mathrm{C}$, were transferred to fresh minimal medium containing $100 \mathrm{~mm}-\mathrm{DHA}$ at $37^{\circ} \mathrm{C}$, no growth was observed. If transferred to $100 \mathrm{~mm}$-DHA at $30^{\circ} \mathrm{C}$ normal growth occurred. Mycelia of glcIl grown at $30^{\circ} \mathrm{C}$ with $100 \mathrm{~mm}$-DHA as carbon source continued to grow when transferred to $37^{\circ} \mathrm{C}$. These transfer experiments indicate that in the glcIl mutant a factor that is necessary for growth on DHA has become temperature sensitive. Once formed at permissive temperature this factor remains active when mycelium is transferred to a non-permissive temperature. This factor is not formed on D-fructose, but requires DHA to be present. In other transfer experiments with $g l c I l$ it has been found that glycerol, but not D-galacturonate, can also induce this factor at permissive temperatures.

A good correlation was found between the induction of glycerol kinase and FAD-dependent G3P dehydrogenase activities and growth of glcIl. In transfer experiments allowing growth of $g l c I l$ on DHA, both enzymes were found at induced levels whereas in non-growing mycelia glycerol kinase could not be detected and FADdependent G3P dehydrogenase activities were lower than $10 \mathrm{mU}$ (mg protein) $)^{-1}$.

\section{Effect of oxygen tension on the glcI phenotype}

Under microaerophilic conditions (see Methods) the glcI mutant did grow, albeit more slowly than the wild-type, on DHA at $37^{\circ} \mathrm{C}$. On solid media containing glycerol as carbon source growth of $g l c I$ was also slower than wildtype WG096 under these conditions. An oxygen concentration effect is also observed with glc $A$ mutant strains, which did not grow on DHA or glycerol under aerobic conditions. Under microaerophilic conditions, however, the glcA mutant grew like the wild-type on solid media containing DHA as carbon source, but it still did not grow on glycerol.

Elevated levels of enzymes at the fructose 6-phosphate level in the glcL1 mutant

Since the $g l c L I$ mutant grew slowly on all carbon sources tested, enzyme levels in mycelia grown in minimal medium containing glucose $(50 \mathrm{mM})$ or acetate $(100 \mathrm{mM})$

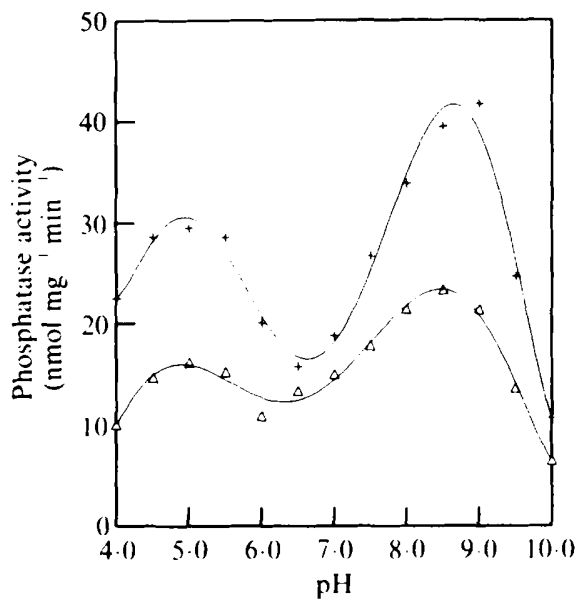

Fig. 2. Intracellular phosphatase activities in $A$. nidulans wild-type $(\triangle)$ and the glcKl mutant $(+)$ grown on minimal medium containing $50 \mathrm{~mm}$-glucose as carbon source. Activities are expressed in nmol p-nitrophenol liberated (mg protein) ${ }^{-1} \min ^{-1}$ at $30^{\circ} \mathrm{C}$. A $10 \mathrm{~mm}$ piperazine/glycylglycine buffer was used at all $\mathrm{pH}$ values.

as carbon source were compared to the activities found in wild-type WG096. Of all enzymes measured (glycolytic, gluconeogenic, pentose phosphate pathway and some enzymes involved in glycerol and mannitol metabolism) only two activities were found to be significantly altered in the glcLl mutant grown on glucose, viz. phosphofructokinase and fructose-6-phosphate reductase. Other enzymes stayed within $40 \%$ deviation from wild-type levels. The phosphofructokinase level was 4.3-fold higher than in the wild-type $\left(3125 \mathrm{mU} \mathrm{mg}^{-1}\right.$ vs. 734

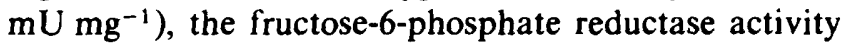
was 6.1 fold higher $\left(4316 \mathrm{mU} \mathrm{mg}^{-1}\right.$ vs. $\left.707 \mathrm{mU} \mathrm{mg}^{-1}\right)$. Grown on acetate, phosphof ructokinase activities were 3.4-fold higher in the $g l c L 1$ mutant $\left(644 \mathrm{mU} \mathrm{mg}^{-1}\right.$ vs. 192

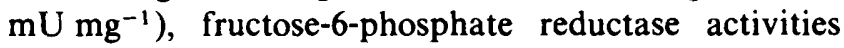
2-2-fold higher $\left(828 \mathrm{mU} \mathrm{mg}^{-1}\right.$ vs. $\left.383 \mathrm{mU} \mathrm{mg}^{-1}\right)$ and pyruvate kinase activities $2 \cdot 1$-fold higher $\left(276 \mathrm{mU} \mathrm{mg}^{-1}\right.$ vs. $130 \mathrm{mU} \mathrm{mg}^{-1}$ ).

\section{Higher intracellular phosphatase activities are found in the glcKI mutant}

Meiotic crosses of $g l c K 1$ strains with tester strain WG362 indicated that the $g l c K$ locus is near to the palc $A$ locus on chromosome II. We addressed the possibility that the glcKl mutant, like palcA mutants (Caddick et al., 1986), has an altered phosphatase regulation. Intracellular phosphatase activities in $g l c K l$ and wild-type mycelium, grown on minimal medium containing glucose as a carbon source, were determined at different $\mathrm{pH}$ values. The phosphatase spectra found are shown in Fig. 2. Both acid and alkaline phosphatase activities were approximately 2-fold higher in the glcKl mutant compared to the wild-type WG096. 


\section{Discussion}

The results presented in this paper indicate that the glcB33 strain is suitable to select pseudorevertants, some of which clearly affect regulation of glycerol metabolism. Since the parental strain has a mutation that leads to G3P accumulation on a mixture of D-glucose and glycerol, pseudorevertants are expected which no longer accumulate G3P or which are less sensitive to G3P accumulation. Two classes of predicted pseudorevertants, following the model for glycerol catabolism proposed by Hondmann et al. (1991), are glycerol uptake $(\mathrm{glcC})$ mutants and glycerol kinase $(\mathrm{glcA})$ mutants. Of the 20 pseudorevertants analysed, twelve were found to be glc $A$ mutants and three were found to be glcC mutants. This confirms that in $A$. nidulans the main route for glycerol catabolism involves an uptake system, encoded by $g l c C$, and substrate phosphorylation by glycerol kinase, encoded by $g l c A$.

Of greatest interest, however, were the two $\mathrm{glcH}$ mutants obtained. In these mutants the enzymes of the aerobic catabolic glycerol pathway are not or are only slightly induced. The low levels of G3P found in these mutants after growth on D-glucose/glycerol are in good agreement with lack of induction. The $\mathrm{glcH}$ locus is located very close to the $g l c A$ locus on chromosome V. It seems reasonable to speculate that the glcH gene could encode a positive regulator required for pathway-specific induction of glycerol catabolism. For several other catabolic pathways in $A$. nidulans it has been established that a gene encoding a positive regulator is clustered with one or more structural genes controlled by it. Wellknown examples of this are the linked alcR (positive regulator) and alcA (alcohol dehydrogenase I) genes involved in ethanol catabolism (Lockington et al., 1985; Felenbok et al., 1988), the genes involved in the utilization of quinic acid, including probably two regulatory genes (Hawkins et al., 1982; Grant et al., 1988) and the genes, including a regulatory gene, necessary for proline utilization (Arst \& MacDonald, 1978; Sharma \& Arst, 1985; Hull et al., 1989). An alternative explanation that accounts for the $\mathrm{glcH}$ characteristics is that the $\mathrm{glcH}$ gene encodes an enzyme which is involved in the synthesis of the co-inducer for the glycerol catabolic genes. Less likely is that the $\mathrm{glcH} 1$ and $\mathrm{glcH} 2$ mutations are in fact mutations in regulatory regions of the glycerol kinase gene, which not only lead to an uninducible glycerol kinase gene, but also impair full induction of one or more of the genes encoding the FADdependent G3P dehydrogenase subunits. In the L-proline catabolism gene cluster of $A$. nidulans, 1 centiMorgan corresponds to a physical distance of 3-4 kb (Hull et al., 1989). If comparable, the distance between $g l c A$ and the
glcH locus would be in the order of $10 \mathrm{~kb}$, thus making this last explanation unlikely to be true.

The accumulation of G3P on glucose/glycerol and glucose/DHA in a glcB33 background and enzymic data indicate that in the glcIl mutant, under aerobic conditions, the uptake systems for glycerol and DHA, and also DHA reductase and glycerol kinase are functioning properly. Under microaerophilic conditions the glcIl mutant does grow on DHA at $37^{\circ} \mathrm{C}$, presumably because the proposed bypass via DHA kinase (Hondmann et al., 1991) can function in the glcIl mutant. Why the glcIl mutant shows a lag-period of several days before growth on DHA at $37^{\circ} \mathrm{C}$ under aerobic conditions starts, remains an unanswered question. Apparently, the $g l c I$ gene encodes a factor that is essential for aerobic growth on DHA. This factor must also be involved in aerobic glycerol catabolism, otherwise a $g l c I l g l c B 33$ strain would not have been found in the selection scheme used. The nature of the selection makes it unlikely that $g l c I$ is a component of a DHA detoxification system.

The glcKl mutant has lower growth rates, higher intracellular phosphatase activities and in a $g l c B 33$ background low G3P levels. This seems to indicate that the accumulation of G3P in the glcB33glcKl strain can be overcome, at least to some extent, although at cost of loss of ATP. In the outcrossed glcKl strain higher levels of intracellular phosphatase activities can account for slower growth rates due to futile cycling of phosphorylated metabolites. Whether the glcKl mutant is, in fact, a palc $A$ mutant, or only maps near to the palc $A$ locus has not been investigated.

The exact nature of the $g l c L I$ mutation remains to be elucidated. A number of speculations can be made. The glcL gene could encode a factor which is essential for the expression of the glycerol kinase gene, and which is also involved in the expression of one or more additional genes. A deficiency of this factor causes slow growth on a wide range of carbon sources and no growth on glycerol, DHA and D-galacturonate. Another possibility is that the glcLI mutation represents a mutation in a gene of which the gene-product becomes less sensitive to G3P accumulation. G3P accumulation in the glcL1glcB33 mutant is not as high as in the glcB33 strain, but this difference might be a consequence of slower metabolism.

\footnotetext{
We thank Rob Vaes for his contributions in this study and Dr Herb Arst for his comments on the manuscript. Peter van de Vondervoort is thanked for his practical suggestions, and Jan Hoogendoorn for the production of Fig. 2. This investigation was supported by the Foundation for Fundamental Biological Research (BION) which is subsidized by the Netherlands Organization for Scientific Research (NWO) (grant no. 811-419-261).
} 


\section{References}

ARST, H. N. \& MacDonald, D. W. (1978). Reduced expression of a distal gene of the prn gene cluster in Aspergillus nidulans: genetic evidence for a dicistronic messenger in an eukaryote. Molecular and General Genetics 163, 17-22.

ARST, H. N., HondmanN, D. H. A. \& Visser, J. (1990). A translocation activating the cryptic nitrogen regulation gene are $B$ inactivates a previously unidentified gene involved in glycerol utilization in Aspergillus nidulans. Molecular and General Genetics 223, 134-137.

Bos, C. J. (1986). Induced mutation and somatic recombination as tools for genetic analysis and breeding of imperfect fungi. $\mathrm{PhD}$ thesis, Agricultural University Wageningen, The Netherlands.

CADDICK, M. X. \& ARST, H. N. (1986). Structural genes for phosphatases in Aspergillus nidulans. Genetical Research 47, 83-91.

Caddick, M. X., Brownlee, A. G. \& Arst, H. N. (1986). Phosphatase regulation in Aspergillus nidulans: responses to nutritional starvation. Genetical Research 47, 93-102.

Clutterbuck, A. J. (1974). Aspergillus nidulans. In Handbook of Genetics, vol. 1, pp. 447-510. Edited by R. C. King. New York: Plenum Press.

Clutterbuck, A. J. (1984). Loci and linkage map of the filamentous fungus Aspergillus nidulans (Eidam) Winter $(n=8)$. Genetic Maps 3, 265-273.

Felenbok, B., Sequeval, D., Mathieu, M., Sibley, S., Gwyne, D. I. \& DAVIES, R. W. (1988). The ethanol regulon in Aspergillus nidulans: characterization and sequence of the positive regulatory alc $R$ gene Gene 73, 217-226.

Grant, S., RoberTs, C. F., Lamb, H. K., Stout, M. \& Hawkins, A. R. (1988). Genetic regulation of the quinic acid utilization ( $Q U T)$ gene cluster in Aspergillus nidulans. Journal of General Microbiology 134 347-358.
Hawkins, A. R., Giles, N. H. \& KinghoRn, J. R. (1982). Genetical and biochemical aspects of quinate breakdown in the filamentous fungus Aspergillus nidulans. Biochemical Genetics 20, 271-286.

Hondmann, D. H. A., Busink, R., Witteveen, C. F. B. \& Visser, J. (1991). Glycerol catabolism in Aspergillus nidulans. Journal of General Microbiology 137, 629-636.

Hull, E. P., Green, P. M., Arst, H. N. \& Scazzocchio, C. (1989). Cloning and physical characterization of the L-proline catabolism gene cluster of Aspergillus nidulans. Molecular Microbiology 3, 553559.

Lockington, R. A., Sealy-Lewis, H. M., Scazzocchio, C. \& Davies, R. W. (1985). Cloning and characterization of the ethanol utilization regulon in Aspergillus nidulans. Gene 33, 137-149.

PAYTON, M. A. (1978). A genetic study of sugar metabolism and transport in Aspergillus nidulans. $\mathrm{PhD}$ thesis, University of Leicester, UK.

Pontecorvo, G., Roper, J. A., Hemmons, L. M., MacDonald, K. D \& Bufton, A. W. J. (1953). The genetics of Aspergillus nidulans. Advances in Genetics 5, 141-239.

SchuUrink, R., Busink, R., Hondmann, D. H. A., Witteveen, C. F B. \& VISSER, J. (1990). Purification and properties of NADP+. dependent glycerol dehydrogenases from Aspergillus nidulans and $A$. niger. Journal of General Microbiology 136, 1043-1050.

SHARMA, K. K. \& ARST, H. N. (1985). The product of the regulatory gene of the proline catabolism gene cluster of Aspergillus nidulans is a positive-acting protein. Current Genetics 9, 299-304.

UITZETTER, J. H. A. A., Bos, C. J. \& VisSER, J. (1986). Characterisation of Aspergillus nidulans mutants in carbon metabolism isolated after Dgalacturonate enrichment. Journal of General Microbiology 132, $1167-1172$.

Visser, J, Van Rooijen, R., Dijkema, C., Swart, K. \& Sealy-Lewis, H. M. (1988). Glycerol uptake mutants of the hyphal fungus Aspergillus nidulans. Journal of General Microbiology 134, 655-659. 